J. DIFFERENTIAL GEOMETRY

76 (2007) 155-175

\title{
COUNTS OF MAPS TO GRASSMANNIANS AND INTERSECTIONS ON THE MODULI SPACE OF BUNDLES
}

\author{
Alina Marian \& Dragos Oprea
}

\begin{abstract}
We show that intersection numbers on the moduli space of stable bundles of coprime rank and degree over a smooth complex curve can be recovered as highest-degree asymptotics in formulas of Vafa-Intriligator type. In particular, we explicitly evaluate all intersection numbers appearing in the Verlinde formula. Our results are in agreement with previous computations of Witten, Jeffrey-Kirwan and Liu. Moreover, we prove the vanishing of certain intersections on a suitable Quot scheme, which can be interpreted as giving equations between counts of maps to the Grassmannian.
\end{abstract}

\section{Introduction}

The Vafa-Intriligator formula counts degree $d$ maps from a smooth complex projective curve $C$ to the Grassmannian $G(r, N)$ of rank $r$ subspaces of the vector space $\mathbb{C}^{N}$, under incidence conditions with special Schubert subvarieties at fixed domain points. When finite, the number of these maps is expressed as the sum of evaluations of a rational function at distinct $N^{\text {th }}$ roots of unity. Such sums are fairly complicated in general, and are not immediately expressible in a compact form. In this paper we show that intersection numbers on the moduli space $\mathcal{N}$ of rank $r$ and degree $d$ stable bundles on $C$, in the case when $r$ and $d$ are relatively prime, arise as coefficients of highest-degree terms in $N$ in Vafa-Intriligator sums. We explicitly compute these leading coefficients as iterated residues. We determine in this way all intersection numbers on $\mathcal{N}$ appearing in formulas of Verlinde type.

Expressions for all top intersections of the cohomology generators on $\mathcal{N}$ were originally written down by Witten as possibly divergent infinite sums over the irreducible representations of $S U(r)$ [W1]. An argument of Szenes identified the convergent Witten sums as iterated residues $[\mathbf{S z}]$. The simplest instances of these top-pairing formulas occur in rank

Received 03/10/2006. 
2 ; they received many algebro-geometric or symplectic proofs. By contrast, the higher rank case is more involved. A symplectic derivation of all of the intersection theory of $\mathcal{N}$ was given by Jeffrey and Kirwan $[\mathbf{J K}]$, with intersection numbers expressed as iterated residues. The Jeffrey-Kirwan approach is very powerful, but it requires careful arguments involving nonabelian localization on singular non-compact moduli spaces. A different approach is due to Liu who obtained, by heat kernel methods, some of the intersection numbers as Witten sums $[\mathbf{L}]$. Another derivation via large level limits of Verlinde-type formulas was hinted at in the last section of $[\mathbf{T W}]$. Finally, results for arbitrary groups were obtained by Meinrenken [Me] via symplectic geometry.

The goal of our paper is to present a surprisingly simple and entirely finite dimensional algebro-geometric derivation of the Witten-SzenesJeffrey-Kirwan-residue formulas. The inspiration for the current work was the connection between the intersection theory of $\mathcal{N}$ and the enumerative geometry of Grassmannians, which emerged several years ago in the mathematics and physics literature $[\mathbf{B D W}][\mathbf{W 2}]$. On the physics side, Witten equates the Verlinde formula giving the dimension of nonabelian (possibly parabolic) theta functions on $C$ with a certain count of maps to a Grassmannian. A precise statement (in the absence of parabolic structures) is given later in this introduction. Witten argues that the Grassmannian nonlinear sigma model and the gauged WZW model of $U(r) / U(r)$, obtained after integrating out matter fields, arise as different large-distance limits of the same physical theory, a gauged linear sigma model. Some of the topological amplitudes of the gauged WZW model, which has a direct link to the Verlinde algebra, and of the Grassmannian sigma model should therefore be closely related. In genus 0, this relation was mathematically established by Agnihotri $[\mathbf{A}]$, who proved that the small quantum cohomology of the Grassmannian $G(r, N)$ is isomorphic to the Verlinde algebra of the unitary group $U(r)$ at level $N-r$. In higher genus, we will extract (roughly half of) the intersection theory of the moduli space of bundles from the count of maps to the Grassmannian. In the limit $N \rightarrow \infty$, it may be possible to relate our method (even if only philosophically) to the more abstract approach pursued in $[\mathbf{T}][\mathbf{T W}]$. Note however that the bounded geometry of our setup lends itself to direct intersection-theoretic calculations, via simpler machinery, avoiding the use of $K$-theory on the moduli stack.

To start, we let $\mathcal{N}$ be the moduli space of rank $r$, degree $d$ stable bundles with fixed determinant on a smooth complex projective curve $C$ of genus $g \geq 2$. We will frequently use the notation $\bar{g}=g-1$. We assume throughout that $r$ and $d$ are coprime. Denote by $\mathcal{V}$ the universal 
bundle on $\mathcal{N} \times C$. We Künneth-decompose the Chern classes

$$
c_{i}(\mathcal{V})=a_{i} \otimes 1+\sum_{j=1}^{2 g} b_{i}^{j} \otimes \delta_{j}+f_{i} \otimes \omega, \quad 1 \leq i \leq r,
$$

with respect to a fixed symplectic basis $1, \delta_{1}, \ldots, \delta_{2 g}, \omega$ of $H^{\star}(C)$. The classes $a_{i}, b_{i}^{j}, f_{i}$ thus obtained are generators for the cohomology of $\mathcal{N}$ $[\mathbf{A B}]$. They are, however, not canonical, since the universal bundle $\mathcal{V}$ is only defined up to tensoring with a line bundle from the base $\mathcal{N}$.

A normalization process is required to kill this ambiguity and obtain invariant classes. To this end, we write $x_{1}, \ldots, x_{r}$ for the Chern roots of $\mathcal{V}$, and let

$$
\bar{x}=\frac{1}{r}\left(x_{1}+\ldots+x_{r}\right), \quad \bar{x}_{i}=x_{i}-\bar{x}, 1 \leq i \leq r .
$$

The $i^{\text {th }}$ symmetric elementary polynomials in the normalized variables are written as

$$
s_{i}\left(\bar{x}_{1}, \ldots, \bar{x}_{r}\right)=\bar{a}_{i} \otimes 1+\sum_{j=1}^{2 g} \bar{b}_{i}^{j} \otimes \delta_{j}+\bar{f}_{i} \otimes \omega, \quad 1 \leq i \leq r .
$$

For instance,

$$
\begin{aligned}
& \bar{f}_{2}=f_{2}-\frac{d(r-1)}{r} a_{1}=\frac{1}{2 r} c_{1}(\mathcal{N}), \\
& \bar{a}_{1}=0, \text { and } \bar{a}_{i}=s_{i}\left(\bar{\theta}_{1}, \ldots, \bar{\theta}_{r}\right),
\end{aligned}
$$

where $\theta_{1}, \ldots, \theta_{r}$ are the Chern roots of $\left.\mathcal{V}\right|_{\mathcal{N} \times \text { point }}$.

We can now explain the main result. Consider the formal variables $\mathrm{X}_{1}, \ldots, \mathrm{X}_{r}$ and $\mathrm{Y}_{1}, \ldots, \mathrm{Y}_{r-1}$ such that

$$
\mathrm{X}_{1}+\ldots+\mathrm{X}_{r}=0
$$

$$
\mathrm{Y}_{1}=\mathrm{X}_{1}-\mathrm{X}_{2}, \ldots, \mathrm{Y}_{r-1}=\mathrm{X}_{r-1}-\mathrm{X}_{r} .
$$

Define moreover the function

$$
\mathrm{L}=\left\{\frac{d}{r}\right\} \mathrm{Y}_{1}+\ldots+\left\{\frac{d(r-1)}{r}\right\} \mathrm{Y}_{r-1},
$$

where \{\} denotes the fractional part.

Theorem 1. For any polynomial $\mathrm{P}$ in the normalized $\bar{a}$-classes, the intersection number

$$
\int_{\mathcal{N}} \exp \left(\bar{f}_{2}\right) \mathrm{P}\left(\bar{a}_{2}, \ldots, \bar{a}_{r}\right)
$$


equals the multiple residue

$$
\begin{aligned}
(-1)^{\bar{g}\left(\begin{array}{c}
r \\
2
\end{array}\right) r^{\bar{g}} \operatorname{Res}_{r-1}=0 \ldots \operatorname{Res}_{1}=0} \frac{1}{e^{\mathrm{Y}_{1}}-1} \cdots & \frac{1}{e^{\mathrm{Y}_{r-1}}-1} \\
\cdot \exp (\mathrm{L}) & \cdot \frac{\mathrm{P}\left(s_{2}(\mathrm{X}), \ldots, s_{r}(\mathrm{X})\right)}{\prod_{i<j}\left(\mathrm{X}_{i}-\mathrm{X}_{j}\right)^{2 \bar{g}}} .
\end{aligned}
$$

Here $s_{k}$ are the elementary symmetric functions. The iterated residue is computed from right to left, at each step keeping all but one of the $\mathrm{Y} s$ fixed.

Theorem 1 will be obtained by exploiting the connection between the intersection theory of $\mathcal{N}$ and that of a suitable compactification of the scheme $\operatorname{Mor}_{d}(C, G(r, N))$ of degree $d$ morphisms from $C$ to the Grassmannian $G(r, N)$. This compactification, the scheme Quot $_{r, d}\left(\mathcal{O}^{N}, C\right)$ constructed by Grothendieck, is the fine moduli space of short exact sequences

$$
0 \rightarrow E \rightarrow \mathcal{O}^{N} \rightarrow F \rightarrow 0,
$$

where $F$ is a degree $d$, rank $r$ quotient sheaf of the trivial bundle. With the aid of the dual universal bundle $\mathcal{E}^{\vee}$ on $\operatorname{Quot}_{r, d}\left(\mathcal{O}^{N}, C\right) \times C$, we obtain, via the Künneth decomposition, cohomology classes denoted by $a, b, f$ :

$$
c_{i}\left(\mathcal{E}^{\vee}\right)=\mathrm{a}_{i} \otimes 1+\sum_{j=1}^{2 g} \mathrm{~b}_{i}^{j} \otimes \delta_{j}+\mathrm{f}_{i}, \quad 1 \leq i \leq r .
$$

When the degree $d$ is large, it was established in $[\mathbf{B D W}]$ that the scheme Quot $_{r, d}\left(\mathcal{O}^{N}, C\right)$ is irreducible, generically smooth of the expected dimension

$$
e=\chi\left(E^{\vee} \otimes F\right)=N d-r(N-r) \bar{g} .
$$

In general, this space may be singular and of the wrong dimension. Nonetheless, Quot ${ }_{r, d}\left(\mathcal{O}^{N}, C\right)$ comes equipped with a canonical perfect obstruction theory, and hence with a virtual fundamental cycle [MO]

$$
\left[\operatorname{Quot}_{r, d}\left(\mathcal{O}^{N}, C\right)\right]^{\text {vir }} \in A_{e}\left(\operatorname{Quot}_{r, d}\left(\mathcal{O}^{N}, C\right)\right) \text {. }
$$

Polynomials in the $\mathrm{a}, \mathrm{b}, \mathrm{f}$-classes can then be evaluated against the virtual fundamental cycle. In particular, the Vafa-Intriligator formula (19) proved in $[\mathbf{S T}],[\mathbf{M O}]$ expresses top intersections of a-classes as sums over $N^{\text {th }}$ roots of unity. The highest order $N$-asymptotics of these intersections on Quot $_{r, d}\left(\mathcal{O}^{N}, C\right)$ yield the formulas of Theorem 1.

Our strategy is most simply illustrated by the computation of

$$
\text { Volume }(\mathcal{N})=\int_{\mathcal{N}} \exp \left(\bar{f}_{2}\right) \text {. }
$$


We determine, as an intersection of $a$-classes on the Quot scheme, the number $\mathrm{M}_{r, d}(N)$ of degree $d$ maps $f: C \rightarrow G(r, N)$ with

$$
N\left(\frac{d}{r}-\bar{g}\right)+r \bar{g}
$$

incidence conditions with general sub-Grassmannians $G(r, N-1) \hookrightarrow$ $G(r, N)$, at fixed domain points. (Here, we assume that $d$ is sufficiently large, and $N$ is divisible by $r$.) The volume of $\mathcal{N}$ is then obtained as the leading coefficient in the $N$-asymptotics of the above count of maps:

$$
\mathrm{M}_{r, d}(N)=\frac{1}{r^{g}} \text { volume }(\mathcal{N}) \cdot N^{r^{2} \bar{g}+1}+\text { lower order terms in } N \text {. }
$$

Note that all intersection numbers appearing in the Verlinde formula are covered by Theorem 1. A derivation of this formula via RiemannRoch is contained in the last section of $[\mathbf{J K}]$; the computation for arbitrary structure groups was pursued in $[\mathbf{B L}]$. Denoting by $\mathcal{L}$ the ample generator of Pic $(\mathcal{N})$, we have $c_{1}(\mathcal{L})=r \bar{f}_{2}$; hence

$$
\chi\left(\mathcal{L}^{s}\right)=\int_{\mathcal{N}} \exp \left(s r \bar{f}_{2}\right) \operatorname{Todd}(\mathcal{N})=\int_{\mathcal{N}} \exp \left((s+1) r \bar{f}_{2}\right) \hat{A}(\mathcal{N}) .
$$

Here, $\hat{A}(\mathcal{N})$ is a polynomial in the $\bar{a}$-classes, which is explicitly given in terms of the Chern roots $\theta_{i}$ as

$$
\hat{A}(\mathcal{N})=\prod_{i<j}\left(\frac{\theta_{i}-\theta_{j}}{2 \sinh \frac{\theta_{i}-\theta_{j}}{2}}\right)^{2 \bar{g}} .
$$

The evaluation of the Verlinde Euler characteristic (8) is immediate from equation (5). The emerging residue answer can be recast effortlessly as an intersection number on $\operatorname{Quot}_{r, d}\left(\mathcal{O}^{r(s+1)}, C\right)$ by a backwards application of the residue formula (31) of Section 3. The result is the following striking equality, derived by physical considerations in [W2]:

$$
\chi\left(\mathcal{L}^{s}\right)=\frac{1}{(s+1)^{g}} \int_{\left[\operatorname{Quot}_{r, d}\left(\mathcal{O}^{r(s+1)}, C\right)\right]^{v i r}} \mathrm{a}_{r}^{s(d-r \bar{g})+d} .
$$

Moreover, when $d$ is large, intersections of a-classes on the Quot scheme have enumerative meaning $[\mathbf{B}]$. In particular, the right-hand-side integral of (9) is the finite count $\mathrm{M}_{r, d}(r(s+1))$ of degree $d$ maps to the Grassmannian $G(r, r(s+1))$ with incidences at fixed $s(d-r \bar{g})+d$ domain points with sub-Grassmannians $G(r, r(s+1)-1) \hookrightarrow G(r, r(s+1))$ in general position.

In a different direction, the argument which gives Theorem 1, combined with a rescaling trick we learned from $[\mathbf{E K}]$, establishes a vanishing result about intersections on Quot, which will be presented in 
the last section of this work. We speculate that the analysis of the $N$ asymptotics of these vanishing intersections should lead to a rederivation of the Pontryagin vanishing on the moduli space of bundles.

The paper is organized as follows. First, we explain the setup of [Ma] which relates intersections on the moduli space of stable bundles to intersections on $\operatorname{Quot}_{r, d}\left(\mathcal{O}^{N}, C\right)$ in the large $N$ regime. Using as starting point the Vafa-Intriligator formula, the relevant evaluations on $\operatorname{Quot}_{r, d}\left(\mathcal{O}^{N}, C\right)$ are cast as iterated residues in Section 3. Upon extracting the appropriate asymptotic coefficients of these residues, we immediately obtain in Section 4 the formulas of Theorem 1. For completeness, we also indicate how the results are expressed as infinite sums indexed by the irreducible representations of $S U(r)$, as in [W1]. Finally, the vanishing of intersections on $\operatorname{Quot}_{r, d}\left(\mathcal{O}^{N}, C\right)$ will be proved in the last section.

\section{Verlinde-type intersections through the Quot scheme}

We start by explaining the setup for the proof of Theorem 1. Traditionally, one studies the intersection theory on the moduli space $\mathcal{N}$ of rank $r$ degree $d$ stable bundles with fixed determinant. However, our computations will be most naturally carried on the moduli space $\mathcal{M}$ of stable bundles $V$ with varying determinant. These intersections will be transfered to $\mathcal{N}$ via the the degree $r^{2 g}$ étale cover

$$
\tau: \mathcal{N} \times J \rightarrow \mathcal{M}
$$

given by tensoring with degree 0 line bundles in the Jacobian $J$ of $C$. When switching from $\mathcal{N}$ to $\mathcal{M}$, we will abusively use the same notations for the universal bundle, its Chern roots and the Künneth components of its Chern classes.

The main technical ingredient of our argument is a precise relationship between the intersection theory of $\mathcal{M}$ and that of $\operatorname{Quot}_{r, d}\left(\mathcal{O}^{N}, C\right)$. We will assume that $d$ is large compared to $N, r$ and $g$ to ensure that Quot $_{r, d}\left(\mathcal{O}^{N}, C\right)$ is irreducible of the expected dimension. We will think of the points

$$
0 \rightarrow E \rightarrow \mathcal{O}^{N} \rightarrow F \rightarrow 0
$$

of $\operatorname{Quot}_{r, d}\left(\mathcal{O}^{N}, C\right)$ as $N$ tuples of sections of the bundle $E^{\vee}$ which generically generate the fibers. Requiring that $V=E^{\vee}$ be stable and not demanding that the sections generically generate, we arrive at a different moduli space, birational to $\operatorname{Quot}_{r, d}\left(\mathcal{O}^{N}, C\right)$, which we denote by $\mathbb{P}_{N, r, d}$. $\mathbb{P}_{N, r, d}$ finely parametrizes pairs $(V, \phi)$ where $V$ is a stable rank $r$ degree $d$ bundle on $C$, and $\phi$ is a non-zero $N$ tuple of holomorphic sections, considered projectively

$$
\phi: \mathcal{O}^{N} \rightarrow V
$$


When $d$ is large, the space $\pi: \mathbb{P}_{N, r, d} \rightarrow \mathcal{M}$ is the projective bundle $\mathbb{P}_{N, r, d}=\mathbb{P}\left(\mathcal{H}^{N}\right)$, where

$$
\mathcal{H}=p r_{\star} \mathcal{V},
$$

with $p r$ denoting the projection from $\mathcal{M} \times C$ to $\mathcal{M}$.

There is a universal morphism

$$
\Phi: \mathcal{O}^{N} \rightarrow \mathcal{U} \text { on } \mathbb{P}_{N, r, d} \times C,
$$

and it is easy to see that

$$
\mathcal{U}=\pi^{\star} \mathcal{V} \otimes \mathcal{O}_{\mathbb{P}}(1)
$$

We Künneth decompose the Chern classes of $\mathcal{U}$ as

$$
c_{i}(\mathcal{U})=\mathrm{a}_{i} \otimes 1+\sum_{j=1}^{2 g} \mathrm{~b}_{i}^{j} \otimes \delta_{j}+\mathrm{f}_{i} \otimes \omega, 1 \leq i \leq r .
$$

The reader who compared (7) and (11) may have noticed the abusive notation. The reason for using the same symbols for analogous, but certainly different classes on $\mathbb{P}_{N, r, d}$ and Quot ${ }_{r, d}\left(\mathcal{O}^{N}, C\right)$, is that both moduli spaces and their universal structures agree on an open subscheme. Usually we will carefully distinguish between the different $a, b, f$-classes, by always mentioning the moduli spaces where the intersections are computed. As before, we let $\overline{\mathrm{a}}_{i}, \overline{\mathrm{b}}_{i}^{j}, \overline{\mathrm{f}}_{i}$ be the normalized classes either on Quot $_{r, d}\left(\mathcal{O}^{N}, C\right)$ or $\mathbb{P}_{N, r, d}$. (Note, however, that in contrast with $\mathcal{M}$, the universal structures on $\operatorname{Quot}_{r, d}\left(\mathcal{O}^{N}, C\right)$ and $\mathbb{P}_{N, r, d}$ are canonical.)

We summarize our setup in the following diagram

$$
\begin{aligned}
& \operatorname{Mor}_{d}(C, G(r, N)) \hookrightarrow \operatorname{Quot}_{r, d}\left(\mathcal{O}^{N}, C\right) \ll \cdots \cdots \mathbb{P}_{N, r, d} \\
& \stackrel{\Downarrow}{\mathcal{M}} \underset{\tau}{\sim} \mathcal{N} \times J
\end{aligned}
$$

Let us consider the intersection product

$$
\int_{\operatorname{Quot}_{r, d}\left(\mathcal{O}^{N}, C\right)} \mathrm{P}\left(\overline{\mathrm{a}}_{2}, \ldots, \overline{\mathrm{a}}_{r}\right) \cdot \mathrm{a}_{r}^{M},
$$

where $\mathrm{P}\left(\overline{\mathrm{a}}_{2}, \ldots, \overline{\mathrm{a}}_{r}\right)$ is a polynomial in the normalized $\overline{\mathrm{a}}$-classes of total weighted degree at most $r^{2} \bar{g}+1$, and $M$ is such that

$$
\operatorname{deg} \mathrm{P}+r M=N d-r(N-r) \bar{g} .
$$

This choice of $M$ is possible since $d$ and $r$ are coprime. In addition to our initial assumption that $d$ is large compared to $N, r$ and $g$, we will require that $N$ be large with respect to $r$ and $g$. This warrants the transfer of certain intersections from $\operatorname{Quot}_{r, d}\left(\mathcal{O}^{N}, C\right)$ to $\mathbb{P}_{N, r, d}$, by making sure that the nonoverlapping loci of $\operatorname{Quot}_{r, d}\left(\mathcal{O}^{N}, C\right)$ and $\mathbb{P}_{N, r, d}$ are avoided. Indeed, when $N$ is large, the main theorem of [Ma] asserts that the class $\mathrm{P}\left(\overline{\mathrm{a}}_{2}, \ldots, \overline{\mathrm{a}}_{r}\right) \cdot \mathrm{a}_{r}^{M}$ evaluates identically on $\operatorname{Quot}_{r, d}\left(\mathcal{O}^{N}, C\right)$ and $\mathbb{P}_{N, r, d}$. It 
is moreover easy to observe, using (10), that the normalized $\bar{a}$-classes on $\mathbb{P}_{N, r, d}$ and $\mathcal{M}$ are related by pullback

$$
\mathrm{P}\left(\overline{\mathrm{a}}_{2}, \ldots, \overline{\mathrm{a}}_{r}\right)=\pi^{\star} \mathrm{P}\left(\bar{a}_{2}, \ldots, \bar{a}_{r}\right) .
$$

Consequently, we have that for $N$ large relative to $r$ and $g$, and $d$ large relative to $N, r$ and $g$,

$$
\begin{aligned}
\int_{\text {Quot }_{r, d}\left(\mathcal{O}^{N}, C\right)} \mathrm{P}\left(\overline{\mathrm{a}}_{2}, \ldots, \overline{\mathrm{a}}_{r}\right) \cdot \mathrm{a}_{r}^{M} & =\int_{\mathbb{P}_{N, r, d}} \mathrm{P}\left(\overline{\mathrm{a}}_{2}, \ldots, \overline{\mathrm{a}}_{r}\right) \cdot \mathrm{a}_{r}^{M} \\
& =\int_{\mathcal{M}} \mathrm{P}\left(\bar{a}_{2}, \ldots, \bar{a}_{r}\right) \cdot \pi_{\star}\left(\mathrm{a}_{r}^{M}\right) .
\end{aligned}
$$

The observation which lies at the heart of the argument for Theorem 1 is that the top-degree term in $N$ of the intersection (12) is closely related to the intersection numbers which are the subject of the theorem. We will therefore study the leading behavior in $N$ of (15). $N$ is, however, assumed to be small compared to the degree $d$. We remove this assumption by observing that all intersections appearing in (15) depend only on $d$ modulo $r$. This is clear for the rightmost intersection by the arguments below, and it follows for the one on $\operatorname{Quot}_{r, d}\left(\mathcal{O}^{N}, C\right)$ from the explicit evaluation via the Vafa-Intriligator formula. Alternatively, we can reduce the degree $d$ on $\operatorname{Quot}_{r, d}\left(\mathcal{O}^{N}, C\right)$ directly, making use of the virtual fundamental class on $\operatorname{Quot}_{r, d}\left(\mathcal{O}^{N}, C\right)$ constructed in [MO] and Theorem 2 there, which compares the virtual cycles for same values of $d$ modulo $r$.

Let us examine the pushforward $\pi_{\star}\left(\mathrm{a}_{r}^{M}\right)$. By (10), the Chern roots of $\mathcal{U}$ restricted to $\mathbb{P}_{N, r, d} \times\{$ point $\}$ equal $\pi^{\star} \theta_{1}+\zeta, \ldots, \pi^{\star} \theta_{r}+\zeta$, with $\theta_{1}, \ldots, \theta_{r}$ being the Chern roots of $\left.\mathcal{V}\right|_{\mathcal{M} \times \text { point }}$, and $\zeta=c_{1}\left(\mathcal{O}_{\mathbb{P}}(1)\right)$. Hence,

$$
\begin{aligned}
\pi_{\star}\left(a_{r}^{M}\right) & =\pi_{\star}\left(\left(\pi^{\star} \theta_{1}+\zeta\right)^{M} \cdot \ldots \cdot\left(\pi^{\star} \theta_{r}+\zeta\right)^{M}\right) \\
& =\sum_{l_{1}, \ldots, l_{r}}\left(\begin{array}{c}
M \\
l_{1}
\end{array}\right) \ldots\left(\begin{array}{c}
M \\
l_{r}
\end{array}\right) \theta_{1}^{l_{1}} \cdot \ldots \cdot \theta_{r}^{l_{r}} \cdot \pi_{\star}\left(\zeta^{r M-|l|}\right) \\
& =\sum_{l_{1}, \ldots, l_{r}}\left(\begin{array}{c}
M \\
l_{1}
\end{array}\right) \ldots\left(\begin{array}{c}
M \\
l_{r}
\end{array}\right) \theta_{1}^{l_{1}} \cdot \ldots \cdot \theta_{r}^{l_{r}} \cdot s_{k}\left(\mathcal{H}^{N}\right),
\end{aligned}
$$

where

$$
k=r M-|l|-(N \operatorname{rank} \mathcal{H}-1)=r^{2} \bar{g}+1-|l|-\operatorname{deg} \mathrm{P} .
$$

The following lemma helps determine the leading term in $N$ of the above expression.

Lemma 1. As $N \rightarrow \infty$, we have

$$
s_{k}\left(\mathcal{H}^{N}\right)=\frac{(-1)^{k}}{k !} c_{1}(\mathcal{H})^{k} \cdot N^{k}+\text { lower order terms in } N .
$$


Proof. Letting $h_{1} \ldots h_{s}$ be the Chern roots of $\mathcal{H}$, we have

$$
\begin{aligned}
s_{k}\left(\mathcal{H}^{N}\right) & =\left[\frac{1}{c(\mathcal{H})^{N}}\right]_{(k)}=\left[\frac{1}{\left(1+h_{1}\right)^{N} \ldots\left(1+h_{s}\right)^{N}}\right]_{(k)} \\
& =\sum_{k_{1}+\ldots+k_{s}=k}\left(\begin{array}{c}
-N \\
k_{1}
\end{array}\right) h_{1}^{k_{1}} \cdot \ldots \cdot\left(\begin{array}{c}
-N \\
k_{s}
\end{array}\right) h_{s}^{k_{s}} \\
& =\sum_{k_{1}+\ldots+k_{s}=k} \frac{(-N)^{k_{1}}}{k_{1} !} h_{1}^{k_{1}} \ldots \frac{(-N)^{k_{s}}}{k_{s} !} h_{s}^{k_{s}}+\text { lower terms in } N \\
& =(-1)^{k} \frac{\left(h_{1}+\ldots+h_{s}\right)^{k}}{k !} \cdot N^{k}+\text { lower order terms in } N \\
& =\frac{(-1)^{k}}{k !} c_{1}(\mathcal{H})^{k} \cdot N^{k}+\text { lower order terms in } N .
\end{aligned}
$$

q.e.d.

Observing that

$$
\left(\begin{array}{c}
M \\
l
\end{array}\right)=\frac{1}{l !}\left(\frac{d}{r}-\bar{g}\right)^{l} N^{l}+\text { lower order terms in } N
$$

and using Lemma 1, we compute that the highest exponent of $N$ in (16) is

$$
\mathrm{e}(\mathrm{P})=r^{2} \bar{g}+1-\operatorname{deg} \mathrm{P} .
$$

The corresponding coefficient equals

$$
\begin{aligned}
& \sum_{l_{1}, \ldots, l_{r}} \frac{\theta_{1}^{l_{1}}}{l_{1} !}\left(\frac{d}{r}-\bar{g}\right)^{l_{1}} \cdot \ldots \cdot \frac{\theta_{r}^{l_{r}}}{l_{r} !}\left(\frac{d}{r}-\bar{g}\right)^{l_{r}} \frac{\left(-c_{1}(\mathcal{H})\right)^{k}}{k !} \\
& =\left[\exp \left(\left(\frac{d}{r}-\bar{g}\right)\left(\theta_{1}+\ldots+\theta_{r}\right)-c_{1}(\mathcal{H})\right)\right]_{(\mathrm{e}(\mathrm{P}))} \\
& =\left[\exp \left(\left(\frac{d}{r}-\bar{g}\right) a_{1}-\left((d-\bar{g}) a_{1}-\sum_{j=1}^{g} b_{1}^{j} b_{1}^{j+g}-f_{2}\right)\right)\right]_{(\mathrm{e}(\mathrm{P}))} \\
& =\left[\exp \left(f_{2}-\frac{d(r-1)}{r} a_{1}+\sum_{j=1}^{g} b_{1}^{j} b_{1}^{j+g}\right)\right]_{(\mathrm{e}(\mathrm{P}))}
\end{aligned}
$$

In the third line, $c_{1}(\mathcal{H})$ is computed by Grothendieck-Riemann-Roch for the projection $p r: \mathcal{M} \times C \rightarrow \mathcal{M}$. 
As a consequence of the above equalities, and of (14), (16), we obtain that (15) takes the form

$$
\begin{array}{r}
N^{\mathrm{e}(\mathrm{P})} \int_{\mathcal{M}} \exp \left(f_{2}-\frac{d(r-1)}{r} a_{1}+\sum_{j=1}^{g} b_{1}^{j} b_{1}^{j+g}\right) \mathrm{P}\left(\bar{a}_{2}, \ldots, \bar{a}_{r}\right) \\
+ \text { lower order terms in } N .
\end{array}
$$

We pull back this expression under $\tau: \mathcal{N} \times J \rightarrow \mathcal{M}$, observing that, since $\mathcal{N}$ is simply connected $[\mathbf{A B}]$, we have

$$
\tau^{\star}\left(\sum_{j=1}^{g} b_{1}^{j} b_{1}^{j+g}\right)=r^{2} \Theta, \text { and } \tau^{\star}\left(f_{2}-\frac{d(r-1)}{r} a_{1}\right)=\bar{f}_{2}-r(r-1) \Theta .
$$

Here $\Theta$ is the theta class on the Jacobian $J$. We find that the highest coefficient above equals

$$
\begin{aligned}
& \frac{1}{r^{2 g}} \int_{\mathcal{N} \times J} \exp \left(\bar{f}_{2}+r \Theta\right) \mathrm{P}\left(\bar{a}_{2}, \ldots, \bar{a}_{r}\right) \\
& =\frac{1}{r^{2 g}} \int_{\mathcal{N}} \exp \left(\bar{f}_{2}\right) \mathrm{P}\left(\bar{a}_{2}, \ldots, \bar{a}_{r}\right) \cdot \int_{J} \exp (r \Theta) \\
& =\frac{1}{r^{g}} \int_{\mathcal{N}} \exp \left(\bar{f}_{2}\right) \mathrm{P}\left(\bar{a}_{2}, \ldots, \bar{a}_{r}\right) .
\end{aligned}
$$

We therefore conclude that

$$
\frac{1}{r^{g}} \int_{\mathcal{N}} \exp \left(\bar{f}_{2}\right) \mathrm{P}\left(\bar{a}_{2}, \ldots, \bar{a}_{r}\right)
$$

is the leading coefficient

$$
\left[N^{r^{2} \bar{g}+1-\operatorname{deg} \mathrm{P}}\right] \int_{\operatorname{Quot}_{r, d}\left(\mathcal{O}^{N}, C\right)} \mathrm{P}\left(\overline{\mathrm{a}}_{2}, \ldots, \overline{\mathrm{a}}_{r}\right) \cdot \mathrm{a}_{r}^{M} .
$$

Here, the brackets denote taking the coefficient of the given power of $N$.

\section{Intersections on the Quot scheme as residues}

In order to calculate (18) and thus complete the proof of Theorem 1, we will make use of the Vafa-Intriligator formula $[\mathbf{S T}],[\mathbf{M O}]$. We will rewrite the resulting expression as a residue, essentially following arguments in $[\mathbf{S z}]$ and $[\mathbf{J K}]$. It will then be easy to extract the leading $N$ coefficient in the next section.

To start, we recall the statement of the Vafa-Intriligator formula.

Assume $\mathrm{A}$ is a symmetric top-degree polynomial in the Chern roots $\mathrm{z}_{1}, \ldots, \mathrm{z}_{r}$ of the dual universal sheaf $\mathcal{E}^{\vee}$ restricted to $\operatorname{Quot}_{r, d}\left(\mathcal{O}^{N}, C\right) \times$ 
\{point\}. Then,

$\int_{\left[\operatorname{Quot}_{r, d}\left(\mathcal{O}^{N}, C\right)\right]^{v i r}} \mathrm{~A}\left(\mathrm{z}_{1}, \ldots, \mathrm{z}_{r}\right)=\mathrm{u} N^{r \bar{g}} \sum_{\lambda_{1}, \ldots, \lambda_{r}} \frac{\mathrm{A}\left(\lambda_{1}, \ldots \lambda_{r}\right)\left(\lambda_{1} \cdots \lambda_{r}\right)^{-\bar{g}}}{\prod_{i<j}\left(\lambda_{i}-\lambda_{j}\right)^{2 \bar{g}}}$.

Here $\left(\lambda_{1}, \ldots, \lambda_{r}\right)$ are ordered tuples of distinct $N^{\text {th }}$ roots of 1 and $\mathrm{u}=$ $(-1)^{\bar{g}\left(\begin{array}{l}r \\ 2\end{array}\right)+d(r-1)}$.

The intersection number appearing in (18) is therefore

$\int_{\text {Quot }_{r, d}} \mathrm{P}\left(\overline{\mathrm{a}}_{2}, \ldots, \overline{\mathrm{a}}_{r}\right) \cdot \mathrm{a}_{r}^{M}=\mathrm{u} N^{r \bar{g}} \cdot \sum_{\lambda_{1}, \ldots, \lambda_{r}} \mathrm{Q}\left(\lambda_{1}, \ldots, \lambda_{r}\right) \cdot \frac{\left(\lambda_{1} \ldots \lambda_{r}\right)^{M-\bar{g}}}{\prod_{i<j}\left(\lambda_{i}-\lambda_{j}\right)^{2 \bar{g}}}$.

The polynomial $\mathrm{Q}$ is obtained from $\mathrm{P}$ using the symmetric functions in the normalized variables $\bar{x}_{1}, \ldots, \bar{x}_{r}$ defined as in (1),

$$
\mathrm{Q}\left(x_{1}, \ldots, x_{r}\right)=\mathrm{P}\left(s_{2}\left(\bar{x}_{1}, \ldots, \bar{x}_{r}\right), \ldots, s_{r}\left(\bar{x}_{1}, \ldots, \bar{x}_{r}\right)\right) .
$$

Note now that by (13),

$$
\operatorname{deg} \mathrm{Q}-r(r-1) \bar{g}+r(M-\bar{g}) \equiv 0 \quad \bmod N,
$$

so each summand of (20) is invariant under rescaling by an $N^{\text {th }}$ root of unity. After rescaling the roots of unity $\lambda_{1}, \ldots, \lambda_{r}$ by $\lambda_{r}^{-1}$, we may assume $\lambda_{r}=1$. This normalization changes the power of $N$ by 1 , to account for each possible value of $\lambda_{r}$. Furthermore, we will allow unordered tuples in the sum (20) at the expense of the prefactor $\frac{1}{r !}$. Setting

$$
\mathrm{R}\left(x_{1}, \ldots, x_{r}\right)=\mathrm{Q}\left(x_{1}, \ldots, x_{r}\right) \cdot \prod_{i<j}\left(x_{i}-x_{j}\right)^{-2 \bar{g}},
$$

we rewrite the right hand side of (20) as

$$
N^{r \bar{g}+1} \frac{\mathrm{u}}{r !} \sum_{\lambda_{1}, \ldots, \lambda_{r-1}} \mathrm{R}\left(\lambda_{1}, \ldots, \lambda_{r-1}, 1\right) \cdot\left(\lambda_{1} \cdots \lambda_{r-1}\right)^{M-\bar{g}} .
$$

Here we sum over tuples of roots of 1 which are pairwise distinct and not equal to 1 .

Further set

$$
\alpha_{1}=\frac{\lambda_{1}}{\lambda_{2}}, \ldots, \alpha_{r-2}=\frac{\lambda_{r-2}}{\lambda_{r-1}}, \alpha_{r-1}=\lambda_{r-1},
$$

so that

$$
\lambda_{1}=\alpha_{1} \cdot \alpha_{2} \cdot \ldots \cdot \alpha_{r-1}, \ldots, \lambda_{i}=\alpha_{i} \cdot \ldots \cdot \alpha_{r-1}, \ldots, \lambda_{r-1}=\alpha_{r-1} .
$$

The sum in (23) becomes

$$
\sum_{\left(\alpha_{1}, \ldots, \alpha_{r-1}\right) \in \mathcal{C}} \mathrm{R}\left(\alpha_{1} \cdots \cdot \alpha_{r-1}, \ldots, \alpha_{r-1}, 1\right) \cdot\left(\alpha_{1} \cdot \alpha_{2}^{2} \cdots \cdots \alpha_{r-1}^{r-1}\right)^{M-\bar{g}}
$$


Here $\mathcal{C}$ is the set of all (arbitrary length) tuples $\left(\alpha_{1}, \ldots, \alpha_{s}\right)$ of $N^{\text {th }}$ roots of 1 such that all products

$$
\alpha_{i} \cdot \alpha_{i+1} \cdots \alpha_{j} \neq 1, \text { for } 1 \leq i \leq j \leq s .
$$

Moreover, let us define the integers $0 \leq m_{i}<N$ such that

$$
i(M-\bar{g}) \equiv m_{i} \quad \bmod N, \quad 1 \leq i \leq r-1 .
$$

Then, we can rewrite $(25)$ as

$$
\sum_{\left(\alpha_{1}, \ldots, \alpha_{r-1}\right) \in \mathcal{C}} \mathrm{R}\left(\alpha_{1} \cdot \ldots \cdot \alpha_{r-1}, \ldots, \alpha_{r-1}, 1\right) \cdot \alpha_{1}^{m_{1}} \cdots \alpha_{r-1}^{m_{r-1}} .
$$

Observe that we have

$$
\frac{m_{i}}{N} \rightarrow\left\{\frac{d i}{r}\right\} \text { as } N \rightarrow \infty
$$

Consider now the meromorphic one-form in the variables $y_{1}, \ldots, y_{r-1}$

$$
\Omega_{1}=\mathrm{R}\left(y_{1} \cdot \ldots \cdot y_{r-1}, \ldots, y_{r-1}, 1\right) \cdot y_{1}^{m_{1}} \cdots y_{r-1}^{m_{r-1}} \cdot \frac{N}{y_{r-1}^{N}-1} \frac{d y_{r-1}}{y_{r-1}} .
$$

We think of the variables $y_{1}, \ldots, y_{r-1}$ as standing in a relation to the original $x_{1}, \ldots, x_{r}$ used in (22) completely similar to the relation that the $\alpha \mathrm{s}$ bear to the original $\lambda \mathrm{s}$. That is,

$$
\frac{x_{i}}{x_{r}}=y_{i} \cdot \ldots \cdot y_{r-1}, \quad 1 \leq i \leq r-1 .
$$

For each fixed $\left(\alpha_{1}, \ldots, \alpha_{r-2}\right) \in \mathcal{C}$, the form $\Omega_{1}\left(\alpha_{1}, \ldots, \alpha_{r-2}, y_{r-1}\right)$ is meromorphic on the projective line, with poles at all the $N^{\text {th }}$ roots of unity, and nowhere else. This step makes essential use of the fact that the exponents $m_{i}$ of the $y_{i}$ are roughly non-zero subunitary fractions of $N$, thus eliminating the possibility of poles at 0 or $\infty$. By the global residue theorem the sum of the residues at the poles is zero.

Note that $\Omega_{1}\left(\alpha_{1}, \ldots, \alpha_{r-2}, y_{r-1}\right)$ has simple poles at the roots $\nu$ such that $\left(\alpha_{1}, \ldots, \alpha_{r-2}, \nu\right) \in \mathcal{C}$, i.e., such that

$$
\nu \neq 1, \nu \neq\left(\alpha_{i} \cdots \alpha_{r-2}\right)^{-1}, 1 \leq i \leq r-2 .
$$

Moreover, the residue of $\Omega_{1}\left(\alpha_{1}, \ldots, \alpha_{r-2}, y_{r-1}\right)$ at $y_{r-1}=\nu$ is

$$
\mathrm{R}\left(\alpha_{1} \cdots \alpha_{r-2} \nu, \ldots, \alpha_{r-2} \nu, \nu, 1\right) \cdot \alpha_{1}^{m_{1}} \cdots \alpha_{r-2}^{m_{r-2}} \cdot \nu^{m_{r-1}} .
$$

As a consequence, the sum in (27) becomes

$$
\begin{aligned}
& \sum_{\left(\alpha_{1}, \ldots, \alpha_{r-1}\right) \in \mathcal{C}} \mathrm{R}\left(\alpha_{1} \cdot \ldots \cdot \alpha_{r-1}, \ldots, \alpha_{r-1}, 1\right) \cdot \alpha_{1}^{m_{1}} \cdots \alpha_{r-1}^{m_{r-1}} \\
=- & \sum_{\left(\alpha_{1}, \ldots, \alpha_{r-2}\right) \in \mathcal{C}}\left(\operatorname{Res}_{y_{r-1}=1} \Omega_{1}+\sum_{i=1}^{r-2} \operatorname{Res}_{y_{r-1}=\left(\alpha_{i} \cdots \alpha_{r-2}\right)^{-1}} \Omega_{1}\right),
\end{aligned}
$$

where we used the shorthand $\Omega_{1}=\Omega_{1}\left(\alpha_{1}, \ldots, \alpha_{r-2}, y_{r-1}\right)$. 
For each $i<r-2$ in the above sum of residues, define the rescaled variables

$$
\begin{aligned}
\widetilde{y}_{r-1} & =\alpha_{i} \cdot \ldots \cdot \alpha_{r-2} \cdot y_{r-1}, \\
\widetilde{\alpha}_{r-2} & =\left(\alpha_{i} \cdot \ldots \cdot \alpha_{r-2}\right)^{-1} \cdot \alpha_{r-2}, \\
\widetilde{\alpha}_{i} & =\left(\alpha_{i} \cdot \ldots \cdot \alpha_{r-2}\right)^{-1} \cdot \alpha_{i} \\
\widetilde{\alpha}_{i-1} & =\left(\alpha_{i} \cdot \ldots \cdot \alpha_{r-2}\right) \cdot \alpha_{i-1} .
\end{aligned}
$$

We keep the rest of the $\alpha$ s unchanged. For $i=r-2$, we use the same rescalings, except that we need to interpret the second and third line as

$$
\widetilde{\alpha}_{r-2}=\alpha_{r-2}^{-1} .
$$

In either case, the rescaled tuple also belongs to $\mathcal{C}$. Although this rescaling may seem puzzling at first, its effect on the $x$ variables (29) in the situation when $y_{j}=\alpha_{j}, 1 \leq j \leq r-2$ is very simple: $x_{j}$ is unaffected for $j \neq i, r-1$, and the rescaled $x_{i}$ and $x_{r-1}$ are the old $x_{r-1}$ and $x_{i}$ respectively. Recalling the definition of $\mathrm{R}$ in (22) we see that $\mathrm{R}$ is symmetric in $x_{1}, \ldots, x_{r-1}$, hence it is unmodified by the interchange of $x_{i}$ and $x_{r-1}$. Furthermore, under this interchange,

$$
{\widetilde{y_{1}}}^{m_{1}} \cdots \widetilde{y}_{r-1}^{m_{r-1}}=y_{1}^{m_{1}} \cdots y_{r-1}^{m_{r-1}} \cdot\left(y_{i} \cdots y_{r-2}\right)^{m_{i-1}-m_{i}+m_{r-1}-m_{r-2}} .
$$

Since

$$
m_{i-1}-m_{i}+m_{r-1}-m_{r-2} \equiv 0 \quad \bmod N
$$

by (26), we deduce that

$$
\Omega_{1}\left(\alpha_{1}, \ldots, \alpha_{r-2}, y_{r-1}\right)=\Omega_{1}\left(\widetilde{\alpha}_{1}, \ldots, \widetilde{\alpha}_{r-2}, \widetilde{y}_{r-1}\right) .
$$

We therefore rewrite the sum over residues as

$$
-(r-1) \sum_{\left(\alpha_{1}, \ldots, \alpha_{r-2}\right) \in \mathcal{C}} \operatorname{Res}_{y_{r-1}=1} \Omega_{1}\left(\alpha_{1}, \ldots, \alpha_{r-2}, y_{r-1}\right) .
$$

Repeating the procedure $r-1$ times we get

$$
\begin{gathered}
\sum_{\left(\alpha_{1}, \ldots, \alpha_{r-1}\right) \in \mathcal{C}} \mathrm{R}\left(\alpha_{1} \cdot \ldots \cdot \alpha_{r-1}, \ldots, \alpha_{r-1}, 1\right) \cdot \alpha_{1}^{m_{1}} \ldots \alpha_{r-1}^{m_{r-1}} \\
=(-1)^{r-1}(r-1) ! \operatorname{Res}_{y_{r-1}=1} \ldots \operatorname{Res}_{y_{1}=1} \Omega_{r-1}\left(y_{1}, \ldots, y_{r-1}\right),
\end{gathered}
$$

where the multiple residue is evaluated from right to left. Here, we defined

$$
\begin{array}{r}
\Omega_{r-1}\left(y_{1}, \ldots, y_{r-1}\right)=\mathrm{R}\left(y_{1} \cdots y_{r-1}, \ldots, y_{r-1}, 1\right) y_{1}^{m_{1}} \cdots y_{r-1}^{m_{r-1}} \\
\cdot \frac{N}{y_{1}^{N}-1} \cdots \frac{N}{y_{r-1}^{N}-1} \frac{d y_{1}}{y_{1}} \cdots \frac{d y_{r-1}}{y_{r-1}} .
\end{array}
$$




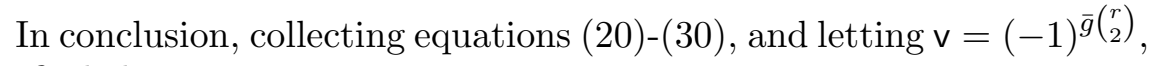
we find that

$$
\int_{\operatorname{Quot}_{r, d}\left(\mathcal{O}^{N}, C\right)} \mathrm{P}\left(\overline{\mathrm{a}}_{2}, \ldots, \overline{\mathrm{a}}_{r}\right) \mathrm{a}_{r}^{M}=\frac{\mathrm{v}}{r} N^{r \bar{g}+1} \cdot \operatorname{Res}_{y_{r-1}=1} \ldots \operatorname{Res}_{y_{1}=1} \Omega_{r-1} .
$$

\section{N-asymptotics of intersections on the Quot scheme and Witten's sums}

4.1. The intersections on the moduli space of bundles. To finish the proof of the main result, we use the conclusions of the previous two sections, in particular formulas (18) and (31). The intersection number

$$
\int_{\mathcal{N}} \exp \left(\bar{f}_{2}\right) \mathrm{P}\left(\bar{a}_{2}, \ldots, \bar{a}_{r}\right)
$$

is obtained as the coefficient of the highest power of $N$ in the expression

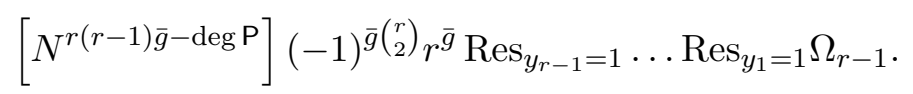

To determine this leading coefficient, we first substitute

$$
y_{i}=\exp \left(\frac{\mathrm{Y}_{i}}{N}\right)=\exp \left(\frac{\mathrm{X}_{i}-\mathrm{X}_{i+1}}{N}\right), 1 \leq i \leq r-1
$$

The variables $\mathrm{X}_{1}, \ldots, \mathrm{X}_{r}$ above are defined by the system of equations (2) and (3). Then, we rewrite the residue in (32) as

$$
\begin{aligned}
& \operatorname{Res}_{r-1}=0 \ldots \operatorname{ResY}_{1}=0 \frac{1}{e^{\mathrm{Y}_{1}}-1} \cdots \frac{1}{e^{\mathrm{Y}_{r-1}-1}} \cdot \mathrm{R}\left(e^{\frac{\mathrm{X}_{1}-\mathrm{X}_{r}}{N}}, \ldots, e^{\frac{\mathrm{X}_{r-1}-\mathrm{X}_{r}}{N}}, 1\right) \\
& \cdot \exp \left(\frac{m_{1}}{N} \mathrm{Y}_{1}+\ldots+\frac{m_{r-1}}{N} \mathrm{Y}_{r-1}\right) \text {. }
\end{aligned}
$$

Note that since $\mathrm{R}$ is in fact a function of the normalized variables $\bar{x}_{1}, \ldots, \bar{x}_{r}$, the $N$-expansion of

$$
\mathrm{R}\left(e^{\frac{\mathrm{x}_{1}-\mathrm{x}_{r}}{N}}, \ldots e^{\frac{\mathrm{x}_{r-1}-\mathrm{x}_{r}}{N}}, 1\right)
$$

starts as

$$
N^{r(r-1) \bar{g}-\operatorname{deg} \mathrm{P}} \mathrm{R}\left(\mathrm{X}_{1}, \ldots, \mathrm{X}_{r}\right)+\text { lower order terms in } N .
$$

Moreover, one sees from (28) that

$$
\begin{aligned}
& \lim _{N \rightarrow \infty}\left(\frac{m_{1}}{N} \mathrm{Y}_{1}+\ldots+\frac{m_{r-1}}{N} \mathrm{Y}_{r-1}\right) \\
& =\left\{\frac{d}{r}\right\} \mathrm{Y}_{1}+\ldots+\left\{\frac{d(r-1)}{r}\right\} \mathrm{Y}_{r-1}=\mathrm{L} .
\end{aligned}
$$

Taking this into account, and using the definition of $\mathrm{R}$ in (21) and (22), we find that the highest power of $N$ in the residue (32) has the 
coefficient

$$
\begin{aligned}
& \operatorname{Res}_{r-1}=0 \cdots \operatorname{Res}_{Y_{1}}=0 \frac{1}{e^{\mathrm{Y}_{1}}-1} \cdots \frac{1}{e^{\mathrm{Y}_{r-1}}-1} \cdot \exp (\mathrm{L}) \\
& \cdot \frac{\mathrm{P}\left(s_{2}\left(\mathrm{X}_{1}, \ldots, \mathrm{X}_{r}\right), \ldots, s_{r}\left(\mathrm{X}_{1}, \ldots, \mathrm{X}_{r}\right)\right)}{\prod_{i<j}\left(\mathrm{X}_{i}-\mathrm{X}_{j}\right)^{2 \bar{g}}} \text {. }
\end{aligned}
$$

The statement of Theorem 1 follows immediately.

Remark 1. One can include the odd cohomology classes $\bar{b}_{i}^{j}$ in the calculation by applying a suitable version of Vafa-Intriligator involving odd b-classes on $\operatorname{Quot}_{r, d}\left(\mathcal{O}^{N}, C\right)$. Such statements are proved, in a particular case, in Proposition 2 of [MO], but the method used there extends in general. To keep the notation as simple as possible, we decided not to write down the general formulas, leaving them to the interested reader. The remaining intersections involving other $f$-classes can be lifted easily to the Quot scheme and can in principle be computed there by equivariant localization as set up in [MO]. We do not yet know of a systematic way of computing all intersections using the methods of this paper, i.e., by studying $N$ asymptotics of the Vafa-Intriligator formula alone.

4.2. Witten's sums. We rewrite the intersections computed in Theorem 1 as infinite sums over the representations of $S U(r)$, which is possible when the degree of $P$ is small enough with respect to the genus $g$. We match the formulas written down in $[\mathbf{W} \mathbf{1}],[\mathbf{L}]$. Note that the arguments of Szenes-Jeffrey-Kirwan also show that the iterated residues reproduce the Witten sums, but here we offer a direct derivation from equation (18), bypassing the residue calculations of Section 3.

Using (18) and (20), we equate

$$
\int_{\mathcal{N}} \exp \left(\bar{f}_{2}\right) \mathrm{P}\left(\bar{a}_{2}, \ldots, \bar{a}_{r}\right)
$$

with the coefficient of $N^{r(r-1) \bar{g}+1-\operatorname{deg} \mathrm{Q}}$ in the sum over unordered tuples

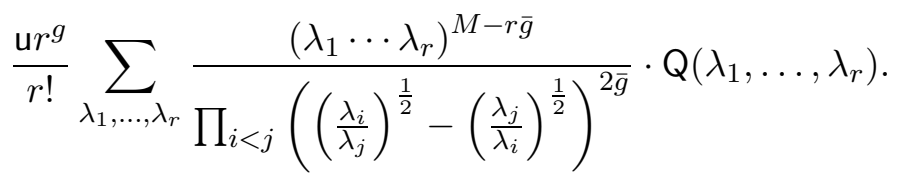

For each product $\lambda_{1} \cdots \lambda_{r}$ there is a unique $\zeta$ such that

$$
\zeta^{r}=\lambda_{1} \cdots \lambda_{r}
$$

and such that, when setting

we have

$$
\nu_{i}=\lambda_{i} \zeta^{-1}=\exp \left(\frac{2 \pi i}{N} \mu_{i}\right)
$$

$$
\mu_{1}+\ldots+\mu_{r}=0 \text { with }-2 N<\mu_{i}-\mu_{j}<2 N \text { integers. }
$$


Then, we compute

$$
\begin{aligned}
\left(\lambda_{1} \cdots \lambda_{r}\right)^{M-r \bar{g}} \mathrm{Q}\left(\lambda_{1} \cdots \lambda_{r}\right) & =\zeta^{r(M-r \bar{g})+\operatorname{deg} \mathrm{Q}} \mathrm{Q}\left(\nu_{1}, \ldots, \nu_{r}\right) \\
& =\zeta^{N(d-r \bar{g})} \mathrm{Q}\left(\nu_{1}, \ldots, \nu_{r}\right) \\
& =\left(\frac{\lambda_{r}}{\nu_{r}}\right)^{N d} \mathrm{Q}\left(\nu_{1}, \ldots, \nu_{r}\right) \\
& =\frac{1}{\nu_{r}^{N d}} \mathrm{Q}\left(\nu_{1}, \ldots, \nu_{r}\right) .
\end{aligned}
$$

It remains to evaluate the coefficient of $N^{r(r-1) \bar{g}-\operatorname{deg} \mathrm{Q}}$ in the expression

$$
\frac{(-1)^{d(r-1)} r^{g}}{2^{2 \bar{g}\left(\begin{array}{l}
r \\
2
\end{array}\right)} r !} \sum_{\mu} \frac{\exp \left(-2 \pi i d \mu_{r}\right)}{\prod_{i<j}\left(\sin \left(\frac{\mu_{i}-\mu_{j}}{N} \pi\right)\right)^{2 \bar{g}}} \cdot \mathrm{Q}\left(e^{\frac{2 \pi i}{N} \mu_{1}}, \ldots, e^{\frac{2 \pi i}{N} \mu_{r}}\right)
$$

Note that a factor of $N$ disappeared in the normalization process to account for each possible value of the product $\lambda_{1} \cdots \lambda_{r}$.

Now,

$$
\begin{aligned}
\mathrm{Q}\left(e^{\frac{2 \pi i}{N} \mu_{1}}, \ldots, e^{\frac{2 \pi i}{N} \mu_{r}}\right)= & \frac{1}{N^{\operatorname{deg} \mathrm{Q}}} \cdot \mathrm{Q}\left(2 \pi i \mu_{1}, \ldots, 2 \pi i \mu_{r}\right) \\
& + \text { lower terms in } N,
\end{aligned}
$$

where the coefficients of the lower order terms in $N$ are polynomials in $\mu$ of smaller degree than $\operatorname{deg}$ P. Additionally,

$$
\frac{1}{\sin ^{2 \bar{g}} x}=\frac{1}{x^{2 \bar{g}}}+\text { lower terms in } x .
$$

Hence, we can evaluate the leading coefficient in (33) to

$$
\frac{(-1)^{d(r-1)} r^{g}}{(2 \pi)^{2 \bar{g}\left(\begin{array}{c}
r \\
2
\end{array}\right) r !}} \cdot \sum_{\mu} \frac{\exp \left(-2 \pi i d \mu_{r}\right)}{\prod_{i<j}\left(\mu_{i}-\mu_{j}\right)^{2 \bar{g}}} \cdot \mathrm{Q}\left(2 \pi i \mu_{1}, \ldots, 2 \pi i \mu_{r}\right) .
$$

The error terms in (34) and (35) do not contribute. Indeed, the first order error terms uniformly dominate the rest, so it suffices to explain that

$$
\frac{1}{N} \cdot \sum_{\mu} \frac{\text { polynomial in } \mu_{1}, \ldots, \mu_{r}}{\prod_{i<j}\left(\mu_{i}-\mu_{j}\right)^{2 \bar{g}-2}} \rightarrow 0 \text { as } N \rightarrow \infty .
$$

This is easy to see by expressing the preceding sum over the $\mu_{i} \mathrm{~s}$ in terms of new variables $\sigma_{i}=\mu_{i}-\mu_{i+1}$. We can uniquely solve for the $\mu$ s using the constraint $\mu_{1}+\ldots+\mu_{r}=0$. When $\operatorname{deg} \mathrm{P}$ is small compared to $g$, the degree of each $\sigma$ in the numerator is small compared to the degree of $\sigma$ appearing in the denominator. In this case, the sum over $\mu$ 's is convergent. The same argument gives the convergence of the infinite sum (33) when $\operatorname{deg} \mathrm{P}$ is small.

We now express the result in terms of the representation theory of $S U(r)$. We write $e_{i}$ for the coordinates on the dual Cartan algebra, and 
agree that $e_{i}-e_{j}, i<j$ are the positive roots of $S U(r)$. Let

$$
\rho=\sum_{i} \frac{r-2 i+1}{2} e_{i}
$$

be half the sum of the positive roots. We order $\mu_{1}>\mu_{2}>\ldots>\mu_{r}$. Setting

$$
\chi_{i}=\mu_{i}-\frac{r-2 i+1}{2},
$$

we have

$$
\chi_{1} \geq \ldots \geq \chi_{r}, \chi_{i}-\chi_{j} \in \mathbb{Z}, \chi_{1}+\ldots+\chi_{r}=0 .
$$

Thus, we can think of

$$
\chi=\chi_{1} e_{1}+\ldots+\chi_{r} e_{r}
$$

as the highest weight of an irreducible representation $\mathcal{R}_{\chi}$ of $S U(r)$. The Weyl dimension formula gives

$$
\operatorname{dim} \mathcal{R}_{\chi}=\prod_{i<j} \frac{\chi_{i}-\chi_{j}+j-i}{i-j}=\prod_{i<j} \frac{\mu_{i}-\mu_{j}}{i-j} .
$$

Moreover, the scalar action of the central element

$$
c=\exp \left(\frac{2 \pi i d}{r}\right) I
$$

has $\chi$-trace

$\operatorname{Trace}_{\chi}(c)=\exp \left(-2 \pi i d \chi_{r}\right) \operatorname{dim} \mathcal{R}_{\chi}=(-1)^{d(r-1)} \exp \left(-2 \pi i d \mu_{r}\right) \operatorname{dim} \mathcal{R}_{\chi}$.

Putting everything together, we see that (36) transforms into the Witten sums

$$
\int_{\mathcal{N}} \exp \left(\bar{f}_{2}\right) \mathrm{P}\left(\bar{a}_{2}, \ldots, \bar{a}_{r}\right)=\mathrm{C} \cdot \sum_{\chi} \frac{\operatorname{Trace}_{\chi}(c)}{\left(\operatorname{dim} \mathcal{R}_{\chi}\right)^{2 g-1}} \cdot \mathrm{Q}(2 \pi i(\chi+\rho)),
$$

when $\operatorname{deg} P$ is small compared to the genus. Here, $Q$ is defined in (21), and we used the constant

$$
\mathrm{C}=\frac{r^{g}}{(2 \pi)^{r(r-1) \bar{g}} \cdot 1 !^{2 \bar{g}} \cdots(r-1) !^{2 \bar{g}}} .
$$

\section{Vanishing of intersections on the Quot scheme}

In this final section, we aim to establish the following vanishing statement:

Proposition 1. Assume that $r$ and $d$ are relatively prime, and $r \geq 2$. Let $\mathrm{P}\left(\overline{\mathrm{a}}_{2}, \ldots, \overline{\mathrm{a}}_{r}\right)$ and $\mathrm{S}\left(\mathrm{a}_{1}, \mathrm{a}_{2}, \ldots, \mathrm{a}_{r}\right)$ be polynomials on $\operatorname{Quot}_{r, d}\left(\mathcal{O}^{N}, C\right)$, 
such that the weighted degree $\operatorname{deg} \mathrm{P}>r(r-1) \bar{g}$, and $\operatorname{deg} \mathrm{P}+\operatorname{deg} \mathrm{S}<\frac{N}{r}$. Then, for all positive integers $M$, we have

$$
\int_{\left[\operatorname{Quot}_{r, d}\left(\mathcal{O}^{N}, C\right)\right]^{v i r}} \mathrm{P}\left(\overline{\mathrm{a}}_{2}, \ldots, \overline{\mathrm{a}}_{r}\right) \cdot \mathrm{S}\left(\mathrm{a}_{1}, \mathrm{a}_{2}, \ldots, \mathrm{a}_{r}\right) \cdot \mathrm{a}_{r}^{M}=0 .
$$

Note that Theorem 5 of [MO] implies that the bound on the degree of $\mathrm{P}$ cannot be lowered.

Proposition 1 gives constraints governing the virtual number of maps from $C$ to the Grassmannian with incidence conditions to special Schubert subvarieties at fixed domain points; as mentioned in the introduction, these numbers are actual counts provided that the degree $d$ is large $[\mathbf{B}]$. The exact equations are obtained by linearity from the $a_{i}$-monomials of the product $\mathrm{P} \cdot \mathrm{S}$, by requiring incidences at distinct domain points for each occurrence of $a_{i}$. It would be interesting to interpret these enumerative constraints geometrically.

Proof. Fix polynomials $\mathrm{P}$ and $\mathrm{S}$ as in the statement of the proposition. For dimension reasons, we may assume

$$
\operatorname{deg} \mathrm{P}+\operatorname{deg} \mathrm{S}+r M=N d-r(N-r) \bar{g} .
$$

Using the Vafa-Intriligator formula, we obtain the following sum over unordered tuples of distinct roots of unity:

$$
\begin{aligned}
& \int_{\left[\operatorname{Quot}_{r, d}\left(\mathcal{O}^{N}, C\right)\right]^{v i r}} \mathrm{P}\left(\overline{\mathrm{a}}_{2}, \ldots, \overline{\mathrm{a}}_{r}\right) \cdot \mathrm{S}\left(\mathrm{a}_{1}, \ldots, \mathrm{a}_{r}\right) \cdot \mathrm{a}_{r}^{M} \\
& =\frac{\mathrm{u} N^{r \bar{g}}}{r !} \sum_{\lambda_{1}, \ldots, \lambda_{r}} \mathrm{R}\left(\lambda_{1}, \ldots, \lambda_{r}\right) \cdot \mathrm{T}\left(\lambda_{1}, \ldots, \lambda_{r}\right) \cdot\left(\lambda_{1} \cdots \lambda_{r}\right)^{M-\bar{g}} .
\end{aligned}
$$

Here $\mathrm{T}$ is the polynomial $\mathrm{S}$ expressed in terms of the Chern roots, and $\mathrm{R}$ is defined as before by equations (21) and (22). We rescale the variables to obtain $\lambda_{r}=1$, and express everything in terms of the $\alpha$ s defined in (24). We are then to prove that

$$
\begin{gathered}
\sum_{\left(\alpha_{1}, \ldots, \alpha_{r-1}\right) \in \mathcal{C}} \mathrm{R}\left(\alpha_{1} \cdot \ldots \cdot \alpha_{r-1}, \ldots, \alpha_{r-1}, 1\right) \mathrm{T}\left(\alpha_{1} \cdot \ldots \cdot \alpha_{r-1}, \ldots, \alpha_{r-1}, 1\right) \\
\cdot \alpha_{1}^{m_{1}} \cdots \alpha_{r-1}^{m_{r-1}}=0 .
\end{gathered}
$$

Here $m_{1}, \ldots, m_{r-1}$ are defined as in (26). The meromorphic form

$$
\begin{array}{r}
\Omega_{r-1}=\mathrm{R}\left(y_{1} \cdots y_{r-1}, \ldots, y_{r-1}, 1\right) \cdot \mathrm{T}\left(y_{1} \cdots y_{r-1}, \ldots, y_{r-1}, 1\right) \\
\cdot y_{1}^{m_{1}} \cdots y_{r-1}^{m_{r-1}} \cdot \frac{N}{y_{1}^{N}-1} \cdots \frac{N}{y_{r-1}^{N}-1} \cdot \frac{d y_{1}}{y_{1}} \cdots \frac{d y_{r-1}}{y_{r-1}}
\end{array}
$$

associated with the above sum has poles only at the $N^{\text {th }}$ roots of 1 . Here, we use the assumption that $r$ and $d$ are coprime, and that

$$
\operatorname{deg} P+\operatorname{deg} S<\frac{N}{r}
$$


to infer that there are no poles at zero and infinity.

By (30), it suffices to show that

$$
\operatorname{Res}_{y_{r-1}=1} \ldots \operatorname{Res}_{y_{1}=1} \Omega_{r-1}\left(y_{1}, \ldots, y_{r-1}\right)=0 .
$$

To prove this, we make use of a trick that we learned from [EK]. Using the auxiliary variable $t$, we substitute

$$
y_{i}=\exp \left(\frac{t \mathrm{Y}_{i}}{N}\right)=\exp \left(\frac{t\left(\mathrm{X}_{i}-\mathrm{X}_{i+1}\right)}{N}\right) .
$$

We show the vanishing of the iterated residue $\operatorname{Res}_{Y_{r-1}}=0 \ldots \operatorname{Res}_{Y_{1}}=0$ of the expression

$$
\begin{aligned}
& \mathrm{R}\left(e^{\frac{t\left(\mathrm{x}_{1}-\mathrm{x}_{r}\right)}{N}}, \ldots, e^{\frac{t\left(\mathrm{x}_{r-1}-\mathrm{x}_{r}\right)}{N}}, 1\right) \mathrm{T}\left(e^{\frac{t\left(\mathrm{x}_{1}-\mathrm{x}_{r}\right)}{N}}, \ldots, e^{\frac{t\left(\mathrm{x}_{r-1}-\mathrm{x}_{r}\right)}{N}}, 1\right) \\
\cdot & \exp \left(\frac{m_{1}}{N} \cdot t \mathrm{Y}_{1}+\ldots+\frac{m_{r-1}}{N} \cdot t \mathrm{Y}_{r-1}\right) \frac{t}{e^{t \mathrm{Y}_{1}}-1} \cdots \frac{t}{e^{t \mathrm{Y}_{r-1}-1}} .
\end{aligned}
$$

We have already observed in Section 4 that the expansion of

$$
\mathrm{R}\left(e^{\frac{t\left(\mathrm{X}_{1}-\mathrm{X}_{r}\right)}{N}}, \ldots, e^{\frac{t\left(\mathrm{x}_{r-1}-\mathrm{X}_{r}\right)}{N}}, 1\right)
$$

takes the form

$$
\left(\frac{t}{N}\right)^{\operatorname{deg} \mathrm{P}-r(r-1) \bar{g}} \mathrm{R}\left(\mathrm{X}_{1}, \ldots, \mathrm{X}_{r}\right)+\text { higher terms in } t .
$$

All other terms in (39) are holomorphic in $t$, hence the order in $t$ of the residue above is at least $\operatorname{deg} \mathrm{P}-r(r-1) \bar{g} \geq 1$. On the other hand, the intersection number this residue computes is independent of $t$, and therefore it must vanish.

q.e.d.

Remark 2. By transferring the intersection (38) to $\mathbb{P}_{N, r, d}$ and pushing it forward to $\mathcal{M}$, Proposition 1 is seen to follow from the vanishing of the Pontryagin ring of $\mathcal{M}$ in degree greater than $r(r-1) \bar{g}$, proved in $[\mathbf{E K}]$.

Conversely, it is tempting to speculate that the vanishing statement (38) implies the vanishing of the Pontryagin ring of $\mathcal{N}$. This is true when $r=3$ as shown below. In general, taking $\mathbf{S}=1$ and considering the highest order term in $N$ of the pushforward along $\pi$ yields

$$
\int_{\mathcal{N}} \exp \left(\bar{f}_{2}\right) \cdot \mathrm{P}\left(\bar{a}_{2}, \ldots, \bar{a}_{r}\right)=0 \text { for } \operatorname{deg} \mathrm{P}>r(r-1) \bar{g} \text {. }
$$

An analysis of the full $N$ asymptotics of the pushforward of $\mathrm{S}\left(\mathrm{a}_{1}, \ldots, \mathrm{a}_{r}\right)$. $\mathrm{a}_{r}^{M}$ may be possible, if cumbersome. To start, one needs to examine the full $N$-asymptotics of the Segre class of Lemma 1 , given by

$$
s\left(\mathcal{H}^{N}\right)=\exp \left(N \cdot \sum_{j}(-1)^{j+1} j ! \operatorname{ch}_{j+1}(\mathcal{H})\right) .
$$


Let us now take $r=3$ and $\mathbf{S}=1$. Using the above expression for the Segre class, and observing that $\operatorname{ch}_{j+1}(\mathcal{H})$ is linear in $\bar{f}_{3}$ when $j \geq 1$, we see that the pushforward $\pi_{\star}\left(a_{3}^{M}\right) \mathrm{P}\left(\bar{a}_{2}, \bar{a}_{3}\right)$ on $\mathcal{N} \times J$ equals

$$
\sum_{k} \frac{1}{r^{2 g}} \cdot \frac{N^{e(\mathrm{P})-k}}{k !} \cdot\left(\bar{f}_{3}^{k} \exp \left(\bar{f}_{2}+r \Theta\right) \mathrm{P}\left(\bar{a}_{2}, \bar{a}_{3}\right)+\text { lower terms in } \bar{f}_{3}\right) \text {. }
$$

Using induction on $k$, we obtain the vanishing of all intersection products

$$
\int_{\mathcal{N}} \bar{f}_{3}^{k} \exp \left(\bar{f}_{2}\right) \mathrm{P}\left(\bar{a}_{2}, \bar{a}_{3}\right)=0
$$

The rank 3 Pontryagin vanishing follows, upon including the odd $b$ classes in the Vafa-Intriligator formula. In higher rank, it would be interesting to see whether the consideration of the full $N$ asymptotics of the pushforwards $\mathrm{S}\left(\mathrm{a}_{1}, \ldots, \mathrm{a}_{r}\right) \cdot \mathrm{a}_{r}^{M}$, for all polynomials $\mathrm{S}$ subject to the assumption of Proposition 1, will inductively give the vanishing of all evaluations

$$
\int_{\mathcal{N}} \bar{f}_{2}^{k_{2}} \cdots \bar{f}_{r}^{k_{r}} \cdot \mathrm{P}\left(\bar{a}_{2}, \ldots, \bar{a}_{r}\right)
$$

\section{References}

[A] S. Agnihotri, Quantum cohomology and the Verlinde algebra, Ph.D. Thesis, Oxford University, 1995.

[AB] M.F. Atiyah \& R. Bott, The Yang-Mills equations over Riemann surfaces, Philos. Trans. Roy. Soc. London Ser. A 308 (1982) 523-615, MR 0702806, Zbl 0509.14014.

[B] A. Bertram, Towards a Schubert Calculus for Maps from a Riemann Surface to a Grassmannian, Internat. J. Math. 5 (1994) 811-825, MR 1298995, Zbl 0823.14038.

[BDW] A. Bertram, G. Daskalopoulos, \& R. Wentworth, Gromov Invariants for Holomorphic Maps from Riemann Surfaces to Grassmannians, J. Amer. Math. Soc. 9 (1996) 529-571, MR 1320154, Zbl 0865.14017.

[BL] J.-M. Bismut \& F. Labourie, Symplectic geometry and the Verlinde formulas, Surveys in differential geometry: differential geometry inspired by string theory 5 (1999) 97-311, MR 1772272, Zbl 0997.53066.

[EK] R. Earl \& F. Kirwan, The Pontryagin rings of moduli spaces of arbitrary rank holomorphic bundles over a Riemann surface, J. London Math. Soc. 60 (1999) 835-846, MR 1753817, Zbl 0965.14011.

[JK] L. Jeffrey \& F. Kirwan, Intersection theory on moduli spaces of holomorphic bundles of arbitrary rank on a Riemann surface, Ann. of Math. (2) 148 (1998) 109-196, MR 1652987, Zbl 0949.14021.

[L] K. Liu, Heat Kernel and moduli space I, II, Math. Res. Lett. 3 (1996) 743-762, Math. Res. Lett. 4 (1997) 569-588, MR 1426532, MR 1470427, Zbl 0871.58014, Zbl 0886.58013.

[Ma] A. Marian, On the intersection theory of Quot schemes and moduli of bundles with sections, arxiv AG/0601275, to appear in J. Reine Angew. Math. 
[MO] A. Marian \& D. Oprea, Virtual intersections on the Quot scheme and the Vafa-Intriligator formula, arxiv AG/0505685, Duke Mathematical Journal.

$[\mathrm{Me}] \quad$ E. Meinrenken, Witten's formulas for intersection pairings on moduli spaces of flat G-bundles, Adv. Math. 197 (2005) 140-197, MR 2166180, Zbl 1085.53076.

[ST] B. Siebert \& G. Tian, On quantum cohomology rings of Fano manifolds and a formula of Vafa and Intriligator, Asian J. Math. 1 (1997) 679-695, MR 1621570, Zbl 0974.14040.

[Sz] A. Szenes, Iterated residues and multiple Bernoulli polynomials, Internat. Math. Res. Notices, 18 (1998) 937-956, MR 1653791, Zbl 0968.11015.

[T] C. Teleman, Borel-Weil-Bott theory on the moduli stack of G-bundles over a curve, Invent Math. 134 (1998), 1-57, MR 1646586, Zbl 0980.14025.

[TW] C. Teleman \& C. Woodward, The Index Formula on the Moduli of G-bundles, arxiv AG/0312154.

[W1] E. Witten, Two-dimensional gauge theories revisited, J. Geom. Phys. 9 (1992) 303-368, MR 1185834, Zbl 0768.53042.

[W2] , The Verlinde algebra and the cohomology of the Grassmannian, Geometry, topology and physics, 357-422, Conf. Proc. Lecture Notes Geom. Topology, IV, Internat. Press, Cambridge, MA, 1995, MR 1358625, Zbl 0863.53054 .

Department of Mathematics

YALE UNIVERSITY

E-mail address: alina.marian@yale.edu

Department of Mathematics

STANFORD UNIVERSITY

E-mail address: oprea@math.stanford.edu 